\title{
A Robust Optimisation Approach using CVaR for Unit Commitment in a Market with Probabilistic Offers
}

\author{
W. A. Bukhsh \\ Institute for Energy and Environment, \\ Department of Electronic and Electrical Engineering, \\ University of Strathclyde, Glasgow, United Kingdom. \\ waqquas.bukhsh@strath.ac.uk
}

\author{
A. Papakonstantinou, P. Pinson \\ Centre for Electric Power and Energy, \\ Department of Electrical Engineering, \\ Technical University of Denmark, Kgs. Lyngby, Denmark \\ \{athpapa,ppin\}@elektro.dtu.dk
}

\begin{abstract}
The large scale integration of renewable energy sources (RES) challenges power system planners and operators alike as it can potentially introduce the need for costly investments in infrastructure. Furthermore, traditional market clearing mechanisms are no longer optimal due to the stochastic nature of RES. This paper presents a risk-aware market clearing strategy for a network with significant shares of RES. We propose an electricity market that embeds the uncertainty brought by wind power and other stochastic renewable sources by accepting probabilistic offers and use a risk measure defined by conditional value-at-risk (CVaR) to evaluate the risk of high re-dispatching cost due to the mis-estimation of renewable energy. The proposed model is simulated on a 39-bus network, whereby it is shown that significant reductions can be achieved by properly managing the risks of mis-estimation of stochastic generation.

Index Terms - conditional value-at-risk; market clearing; optimal power flow; risk analysis.
\end{abstract}

\begin{tabular}{cl} 
Sets & \multicolumn{1}{c}{ NomENCLATURE } \\
$\mathcal{B}$ & Buses, indexed by $b$. \\
$\mathcal{L}$ & Lines (edges), indexed by $l$. \\
$\mathcal{G}$ & Generators, indexed by $g$. \\
$\mathcal{W}$ & Wind generators, indexed by $w$. \\
$\mathcal{D}$ & Demands, indexed by $d$. \\
$\mathcal{B}_{l}$ & Buses connected by line $l$. \\
$\mathcal{L}_{b}$ & Lines connected to bus $b$. \\
$\mathcal{G}_{b}$ & Generators located at bus $b$. \\
$\mathcal{D}_{b}$ & Loads located at bus $b$. \\
$\mathcal{S}_{b}$ & Scenarios, indexed by $s$.
\end{tabular}

Parameters

$\begin{array}{ll}b_{l} & \text { Susceptance of line } l . \\ \tau_{l} & \text { Off-nominal tap ratio of line } l . \\ P_{g}^{\mathrm{G}-}, P_{g}^{\mathrm{G}+} & \text { Min., max. real power outputs of conventional } \\ & \text { generator } g . \\ P_{d}^{\mathrm{D}} & \text { Real power demand of load } d . \\ f_{g}\left(p_{g}^{\mathrm{G}}\right) & \text { Cost function for generator } g . \\ \gamma & \text { Prescribed probability level. } \\ C_{w}^{\mathrm{PP}} & \text { Purchase price at node } w . \\ C_{g}^{\mathrm{S}} & \text { Cost of committing generator at bus } g . \\ R_{g}^{ \pm} & \text {Min./max. regulation of generator } g .\end{array}$

$$
\begin{array}{ll}
P_{w}^{\mathrm{W}+} & \text { Max. real power generation from wind farm } w . \\
\Delta P_{w, s}^{\mathrm{W}} & \text { Change in wind power generation forecast. } \\
\eta & \text { Threshold level for the loss function. } \\
\omega & \text { Weighting on the risk measured as defined by } \\
& \text { CVaR. }
\end{array}
$$

Variables

$$
\begin{array}{ll}
p_{g}^{\mathrm{G}} & \text { Real power output of generator } g . \\
p_{w, s}^{\mathrm{W}} & \text { Real power output of wind generator } w . \\
\theta_{b, s} & \text { Voltage phase angle at bus } b . \\
p_{l, s}^{\mathrm{L}} & \text { Real power injection at bus } b \text { into line } l \text { (which } \\
& \text { connects buses } b \text { and } b^{\prime} \text { ). } \\
\Delta P_{g, s}^{\mathrm{G}} & \text { Regulation of conventional generator } g . \\
\Delta P_{g, s}^{\mathrm{G} \pm} & \text { Upward/downward regulation of conventional gen- } \\
& \text { erator } g . \\
u_{g} & \text { Unit commitment variable for generator } g .
\end{array}
$$

\section{INTRODUCTION}

Modern power systems are in a midst of a comprehensive change, primarily driven by the liberalisation of electricity markets and an increased focus on renewable energy sources (RES). Over the last decade, there has been a substantial increase in the installed capacities of RES challenging practices in both transmission system planning and operation [1], as well as in the electricity markets, given that they were designed under the domination of dispatchable and fully predictable sources of energy [2]. Consequently, the support mechanisms that were put in electricity markets to safeguard stochastic producers from the price volatility brought by the intermittent and uncertain nature of RES tend to become inefficient as shares of RES increase and imbalance costs are transferred to the consumers [3], [4]. Such developments highlight the need for electricity markets that embed the very nature of RES in the market mechanism itself, first by replacing deterministic offers (e.g. point forecasts) with probabilistic estimates (e.g. quantiles), and secondly by including risk-aware dispatching mechanisms that are robust enough to accommodate the underlying uncertainties inherent to modern power systems.

In this context, there has been significant academic interest in addressing several of the challenges brought by RES. Most of the day-ahead clearing optimisation models are posed as 
two-stage stochastic programming problems [5], where often the first stage of the problem is to schedule conventional generators and second stage realises the uncertainties from RES. Authors in [6] consider the deployment of reserve capacity in their stochastic programming model and propose an energy-only settlement where the capacity is 'converted' to energy through the market mechanism. One limitation of such approach is that the participants can speculate and therefore influence the market clearing mechanism. This issue is addressed by [7] where the authors propose a single auction that clears the market and arranges the financial settlement, which may require the flexible generators to accept losses for some wind power production realisation. Furthermore, the aforementioned stochastic optimisation approaches that minimise expectation, are risk-neutral. However, for some cases it is important to model risk-averse or risk-taker policies, specifically when operational uncertainty is considered.

In this context, conditional value-at-risk $(\mathrm{CVaR})$ is a risk measure that captures the variability of risk and can allow to implement different risk policies. Authors in [8] propose a stochastic optimal power flow (OPF) model that gives optimal policies regarding scheduling of controllable devices within a power network based on chance constraints. Solutions depend on how the probabilistic constraints are formulated with the model relying on access to the variance of forecast errors. This is partially addressed in [9] with a more straightforward implementation of $\mathrm{CVaR}$, where a stochastic OPF model captures risks of demand-generation imbalances caused by wind power using the risk measure CVaR. The model is based on DC power flow, while unit commitment (UC) is not considered as part of the problem. Both approaches in [8], [9] contribute on the overall discussion, however they face some limitations: i) risk is only associated to a part of operational uncertainty and ii) they are based on conventional market clearing mechanisms using only deterministic offers.

In this paper, we address the existing limitations by proposing a model that produces a robust optimal policy consisting of day-ahead generation schedules that minimise the total cost of generation. Furthermore, the proposed model manages the risk of imbalances due to stochastic generation with stochastic producers' offers modelled as probabilistic estimates, instead of point forecasts, following [10]. In doing so, we shift towards a more realistic model whereby a market can extract the inherently imperfect estimates of stochastic production, instead of relying on transmission system operators' (TSOs') presumed perfect estimates.

We contribute to the state of the art by proposing a two-stage stochastic programming formulation that takes into consideration the effects of unit commitment decisions on the optimal policy, while using a CVaR risk measure in a market where stochastic producers report probabilistic offers. The proposed formulation is tractable as it can account for a large number of scenarios for the future power system operation. Finally, we give insights into questions about risk measures and modelling details including network constraints and technical restriction and test the proposed model on a 39-bus network with real world data from RES. Through numerical simulations we demonstrate the impact of inaccurate forecasts of low predictive value, while we show that by carefully managing the overall risk of system, a more robust policy can be obtained.

The rest of the paper is organised as follows: In Section III we define uncertainty and risk management in the context of electricity markets, while Section III introduces the general formulation, described in detail in Section IV In Section V we numerically evaluate the proposed model and lastly in Section VI we conclude and give future research directions.

\section{UNCERTAINTY AND RISK ASSESSMENT IN ELECTRICITY MARKETS}

In this section, we provide a general framework regarding uncertainty and risk assessment in electricity markets. In Section II-A, we model uncertainty in stochastic production, while in Section II-B we describe the bidding process in a market designed to accommodate probabilistic offers. Finally, in Section II-C we formally define the risk measure in consistence with literature [8].

\section{A. Uncertain production}

Let a stochastic producer, such as a wind farm, face an upper limit $P_{w}^{W+}$ in its output, defined by the specific technical specifications of the deployed wind farm units $w$ and let the real-time generation $p_{w, s, t}^{W}$ be equal to $y_{w} P_{w}^{W+}$, where $y_{w} \in[0,1]$ is a realisation of the random variable $Y$ which models the producer's stochastic output. The variable $Y$ follows a distribution $G$ defined by a set of parameters $\theta$ s.t. $Y \sim G(y ; \theta)$, where $\theta$ can be equal to mean and variance depending on the definition of the used distribution.

\section{B. Modelling probabilistic offers from stochastic producers}

We consider an electricity market that accepts probabilistic offers from the stochastic producers, instead of the deterministic offers e.g. point forecasts. Based on the stochastic framework introduced in [10], producers are asked to submit their predictive distributions that in essence are estimates of distribution $G$. Stochastic producers can report a CDF, a set of quantiles, or in case of a parametric distributions producers can report the parameters of a known distribution. Let $\widehat{\theta}_{w}$ be the set of reported parameters during the bidding stage of a day-ahead market. The parameter set $\widehat{\theta}_{w}$ defines distribution $F(y ; \widehat{\theta})$. It should be noted that $G$ may not necessarily be equal to $F$, as $F$ represents the predictive distribution and $G$ the actual distribution of the stochastic output. In terms of the parameter sets, let $\widehat{\theta}_{w}=\epsilon \theta_{w}$, with $\epsilon$ representing the imperfect nature of an estimate. This parameter demonstrates the impact of possible errors in estimation or possible strategic behaviour on behalf of the producers.

The use of predictive distributions has a significant influence on the optimal solution of a two-stage stochastic model of the day-ahead market. The finite set of scenarios that models stochastic production is sampled from the predictive distribution $F$ as it is the only distribution available to the market 
and system operators prior to the actual production. Given this intrinsic link between the day-ahead schedule and expected balancing stage it becomes clear that the reported offers in the day-ahead market can heavily influence the optimal policy.

\section{Risk measures}

Due to the use of probabilistic offers, it is important for a market operator to derive a summary statistic from the predictive distribution and clear the day-ahead market based on it. In this paper, we assume that this statistic is the mean which the market operator can either extract from the reported CDF, the empirical CDF derived from the set of quantiles given that $\mu=\int_{0}^{\infty}(1-F(t)) d t$, or just by using the reported value in case of a parametric predictive distributions. The selection of the mean is a necessary link with the existing electricity market setup where it is a common practice for stochastic producers to use point forecasts that correspond to conditional expectation estimates [11].

Let $\mu_{w} P_{w}^{\mathrm{W}+}$ be the summary statistic used to clear the dayahead market and let $\Delta P_{w, s}^{\mathrm{W}+}$ be the change in the generation availability corresponding to the scenario $s$ for the generator $w$ in the time period $t$, respectively. The wind power output for the generator $w$ is modelled as follows:

$$
0 \leq p_{w, s}^{\mathrm{W}} \leq P_{w}^{\mathrm{W}+}+\Delta P_{w, s}^{\mathrm{W}+}
$$

Now, integration of renewable energy sources, especially wind power, requires careful assessment of risk when it comes to commitment of conventional power plants. Normally TSOs er towards risk averse policy. They tend to commit more conventional generation than required in order to account for potential power imbalances [12]. However such a risk averse policy is not optimal as it may result in large amounts of renewable energy being curtailed when it comes to clearing the market in the real-time. Therefore there is a need for a risk-aware policy that takes into account the uncertainties from the stochastic producers and optimally manages the risk and rewards in a power system. Risk measures like value-atrisk (Var) and CVaR are two risk assessment measures that are used largely in the financial industry to manage assets. It is commonly known that $\mathrm{CVaR}$ is a superior measure for risk when compared to $\mathrm{VaR}$ [13]. Given a cost function $L(x, \xi): X \times \Xi \rightarrow \mathbb{R}$, the $\gamma-\mathrm{CVaR}$ is:

$$
\phi_{\gamma}(x)=\frac{1}{1-\gamma} \int_{L(x, \xi) \geq \eta_{\gamma}(x)} L(x, \xi) p(\xi) d \xi
$$

where $\eta_{\gamma}(x)$ is $\gamma-\mathrm{VaR}$.

Calculation of $\gamma-\mathrm{CVaR}$ is equivalent to minimisation of the following function:

$$
F_{\gamma}(x, \eta)=\eta+\frac{1}{1-\gamma} \int_{\xi \in \Xi}[L(x, \xi)-\eta]^{+} p(\xi) d \xi
$$

Let the cost function be a linear function describing the cost of real power purchased at a spot market. The above equation can then be approximated such that:

$$
\hat{F}_{\gamma}\left(p_{w}^{\mathrm{W}}, \eta\right)=\eta+\frac{1}{1-\gamma} \mathbb{E}\left[\sum_{w \in \mathcal{W}} C_{w}^{\mathrm{PP}}\left[P_{w}^{\mathrm{W}}-p_{w, s}^{\mathrm{W}}\right]-\eta\right]^{+}
$$

where $C_{w}^{\mathrm{PP}}$ is the purchase price of power at the bus $w$.

\section{CONCEPTUAL FRAMEWORK}

Our conceptual framework is as follows: a TSO has to determine the commitment of conventional power plants. There is a great level of uncertainty from generation from the wind power producers that will obviously effect the commitment of conventional power plants. In this situation, a transmission system needs to find a decision that optimally balances the risks of over-committing expensive conventional generation versus under-utilisation of low cost and clean generation from wind power and other stochastic producers.

We model this problem as a two-stage stochastic programming problem. The TSO has full access to producers' probabilistic offers, publicly available from the market operator and can now measure the risk of extreme a-priori using a regulariser defined in terms of the $\operatorname{CVaR}\left(\hat{F}_{\gamma}\left(p^{\mathrm{w}}, \eta\right)\right.$ in Section II-C). Through this model we investigate if the mean of the scenarios is a good estimate to use in the day-ahead market and determine the appropriate risk level.

The general form of a two-stage stochastic program [5] is

$$
\min c^{\mathrm{T}} x+\mathbb{E}_{\xi} Q(x, \xi)
$$

subject to

$$
\begin{gathered}
A x=b, \\
x \geq 0,
\end{gathered}
$$

where $x$ is a vector of decision variables and $Q(x, \xi)=$ $\min \left\{q^{\mathrm{T}} y: W y=h-T x, y \geq 0\right\}$ is the optimal value of the second stage problem.

The first stage decision variables determine which conventional generator will come online for the day-ahead operation of the power system along with their operating points and these decisions are represented by a vector $x$. In the second stage of the problem, full information is received on realisation of the random process determined by the vector $\xi$, and corrective actions are taken. Corrective actions for the day-ahead decisions are rescheduling of conventional generators and curtailment from the wind power producers. These corrective actions are modelled as a recourse actions: affecting the values of first stage variables and hence influence the objective function of the problem. In next section we give the mathematical formulation of the problem.

\section{Problem Formulation}

Consider a power network with the set of buses $\mathcal{B}$. Let $\mathcal{G}$ be the set of conventional power plants and $\mathcal{S}$ be the set of scenarios from the wind power producers. The constraints and objective function of our optimisation problem are 


\section{A. Power flow}

Let $p_{g}^{\mathrm{G}}$ be the real power generation from the conventional generator $g$. The power balance equations are given as, $\forall b \in$ $\mathcal{B}, s \in \mathcal{S}:$

$$
\sum_{g \in \mathcal{G}_{b}}\left(p_{g}^{\mathrm{G}}+\Delta p_{g, s}^{\mathrm{G}}\right)+\sum_{w \in \mathcal{W}_{b}} p_{w, s}^{\mathrm{W}}=\sum_{d \in \mathcal{D}_{b}} P_{d}^{\mathrm{D}}+\sum_{l \in \mathcal{L}_{b}} p_{l, s}^{\mathrm{L}}
$$

where $p_{w, s}^{\mathrm{W}}$ denotes the real power bid from the renewable generator $w, P_{d}^{\mathrm{D}}$ denotes the real power demand $d$ and $p_{l, s}^{\mathrm{L}}$ is the flow of real power in the line $l$ in scenario $s$, respectively. The power flow equations are given as, $\forall l \in \mathcal{L}, \forall s \in \mathcal{S}$ :

$$
p_{l, s}^{\mathrm{L}}=-\frac{b_{l}}{\tau_{l}}\left(\theta_{b, s}-\theta_{b^{\prime}, s}\right)
$$

where $b$ and $b^{\prime}$ are the two ends of the line $l$. Voltage angles at the two ends of the line $l=\left(b, b^{\prime}\right)$ are denoted by $\theta_{b, s}$ and $\theta_{b^{\prime}, s}$, respectively. We consider the DC model of power flow [14]. The second stage recourse variables $\Delta p_{g, s}^{\mathrm{G}}$ in $(6)$ are modelled in terms of the upward and the downward regulation variables s.t.

$$
\begin{gathered}
\Delta p_{g, s}^{\mathrm{G}}=\Delta p_{g, s}^{\mathrm{G}+}-\Delta p_{g, s}^{\mathrm{G}-} \\
0 \leq \Delta p_{g, s}^{\mathrm{G}+} \leq R_{g}^{+} \\
0 \leq \Delta p_{g, s}^{\mathrm{G}-} \leq R_{g}^{-}
\end{gathered}
$$

where $R_{g, t}^{+}, R_{g, t}^{-}$are the permissible upward and downward regulation of the generator $g$ in the time period $t$, respectively.

\section{B. Unit commitment}

Given the single-dimensional time framework we employ in this research, the unit commitment constraints are straightforward. Specifically, let $u_{g}$, the unit commitment status of generator $g$, be equal to 1 if the generator is online and is 0 otherwise. Furthermore, generation from the conventional generators is bounded by the following inequality constraints:

$$
u_{g} P_{g}^{\mathrm{G}-} \leq p_{g}^{\mathrm{G}} \leq P_{g}^{\mathrm{G}+} u_{g}
$$

where $P_{g}^{\mathrm{G}-}, P_{g}^{\mathrm{G}+}$ are the lower and the upper bounds on the generation output of the generator $g$, respectively.

\section{Operating constraints}

The line flow limits are given by the following set of constraints: $\forall l \in \mathcal{L}$

$$
-P_{l}^{\max } \leq p_{l, s}^{\mathrm{L}} \leq P_{l}^{\max }
$$

where $P_{l}^{\max }$ is the real power capacity limit of the line $l$.

\section{Objective function}

Let $\lambda_{w, s}$ be the probability of the scenario $s$ for the renewable generator $w$. The objective is to minimise the cost of generation from the conventional generators, and optimally utilise the generation from the RES while initiating the demand response from the distribution system operators. Note that we do not consider ramping cost of the generators between the time intervals. Day-ahead cost is given as:

$$
C_{g}^{\mathrm{DA}}\left(p_{g}^{\mathrm{G}}\right)=f\left(p_{g}^{\mathrm{G}}\right)+C_{g}^{\mathrm{S}} u_{g}
$$

where $f\left(p_{g}^{\mathrm{G}}\right)$ is cost of generation and $C_{g}^{\mathrm{S}}$ is the cost of committing generator $g$. Close to real-time operation the system operator has an improved estimate of the actual generation from stochastic sources and the generator may need to get regulated in order to meet the demand. The cost of such regulation is given by the following equation:

$$
C_{g}^{\mathrm{REG}}\left(\Delta p_{g, s}^{\mathrm{G}}\right)=\left(C_{g}^{\mathrm{R}+} \Delta p_{g, s}^{\mathrm{G}+}+C_{g}^{\mathrm{R}-} \Delta p_{g, s}^{\mathrm{G}-}\right)
$$

where $C_{g}^{\mathrm{R}+}$ is the up-regulation cost and $C_{g}^{\mathrm{R}-}$ is the downregulation cost of generator $g$ respectively. The overall objective function of the proposed optimisation model is as follows:

$$
z=\sum_{g \in \mathcal{G}} C_{g}^{\mathrm{DA}}\left(p_{g}^{\mathrm{G}}\right)+\mathbb{E}\left(C_{g}^{\mathrm{REG}}\left(\Delta p_{g, s}^{\mathrm{G}}\right)\right)+\omega \sum_{w \in \mathcal{W}} \hat{F}_{\beta}\left(p_{w}^{\mathrm{W}}, \eta\right)
$$

where $\omega$ is the weighting on the risk measure, $\hat{F}_{\beta}$ is a risk measure as defined in the equation (4).

\section{E. Overall formulation}

The overall formulation of the problem is given as follows:

$$
\min z\left(p_{g}^{\mathrm{G}}, p_{w, s}^{\mathrm{W}}, \Delta p_{g, s}^{\mathrm{G}}\right)
$$

subject to

$$
\begin{array}{l|l|l|l|}
\hline 1 & 4 & 6 & -10 \\
\hline
\end{array}
$$

where $\left(p_{g}^{\mathrm{G}}, p_{w, s}^{\mathrm{W}}, \Delta p_{g, s}^{\mathrm{G}}\right)$ are the decision variables. Depending on the objective function $f\left(p_{g, t}^{\mathrm{G}}\right)$, the overall problem is a linear or a quadratic program (LP or QP). We use CPLEX $12.06[15]$ called from a PYOMO [16] model to solve the problem.

\section{NUMERICAL RESULTS}

In this section, we simulate the proposed model on a 39-bus test network derived from the New England test case in [17], and modified as shown in Fig. 2. In this context, three fossil fuel generators at buses 33, 34 and 36 are replaced by wind generation with twice the capacity of original generation and more realistic costs where derived from [18]. With these modifications the conventional generation accounts for $63 \%$ of the total capacity of the generation in the network. Furthermore, following common practice in literature (c.f. [19]), the cost of up-regulation is assumed to be $10 \%$ higher than the day-ahead cost of generation and cost of down-regulation is $9 \%$ less than the day-ahead cost of generation. The risk quantile, $\gamma$, is equal to 0.95 and 50 scenarios are considered for the experiments, unless otherwise stated.

In our simulations, we assume zero cost of generation from wind power [19]. Moreover wind power from the producer $w$ can be curtailed continuously to zero at the price of $C_{w}^{\mathrm{W}}$. Wind power production uncertainty is modelled by a Beta distribution in consistence with the related literature (cf. 


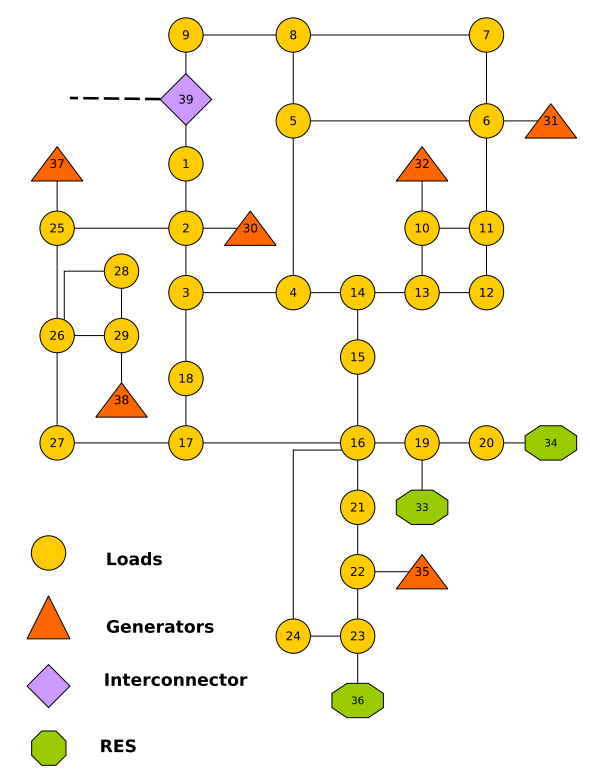

Fig. 1. Modified 39 bus system with 6 conventional generators and 3 wind power producers.

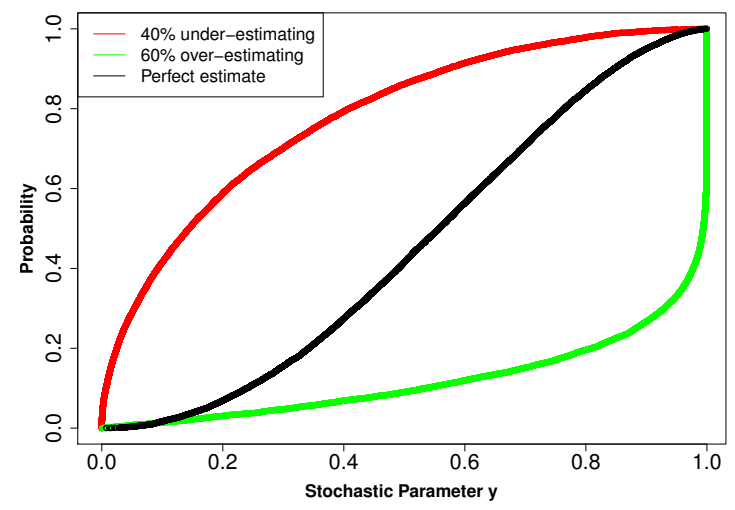

Fig. 2. CDFs for imperfect and perfect estimation

[19], [20]), without this restricting our assumptions regarding uncertainty and risk measurement in Section [II and the theoretical framework in Section IV] Beta distribution that we use for the experiments is defined by mean and variance s.t. $\left(\mu_{w}, \sigma_{w}^{2}\right)=(0.55,0.05)$ with parameters $\alpha_{w}$ and $\beta_{w}$ defined as:

$$
\alpha=\frac{(1-\mu) \mu^{2}}{\sigma^{2}}-\mu, \quad \beta=\frac{(1-\mu) a}{\mu}
$$

In the proposed day-ahead market setup, wind power producers report parameters $\left(\widehat{\mu}_{w}, \widehat{\sigma}_{w}^{2}\right)$ and it is entirely possible that both mean and variance are mis-estimated i.e. $\left(\widehat{\mu}_{w}, \widehat{\sigma}_{w}^{2}\right) \neq\left(\mu_{w}, \sigma_{w}^{2}\right)$, with $\left(\widehat{\mu}_{w}, \widehat{\sigma}_{w}^{2}\right)$. We simplify our analysis by assuming that only the wind power producer located in bus 34 may mis-estimate its parameters and by only considering the mis-estimation of the mean. This translates to $\left(\widehat{\mu}_{w}, \widehat{\sigma}_{w}^{2}\right)=\left(\epsilon \mu_{w}, \sigma_{w}^{2}\right)$ with $\epsilon \in[0.6,0.7, \cdots, 1.3,1.4]$ being a parameter which denotes the imperfect nature of the estimate. Values less than 1 represent under-estimation of the mean, and values above 1 represent over-estimation

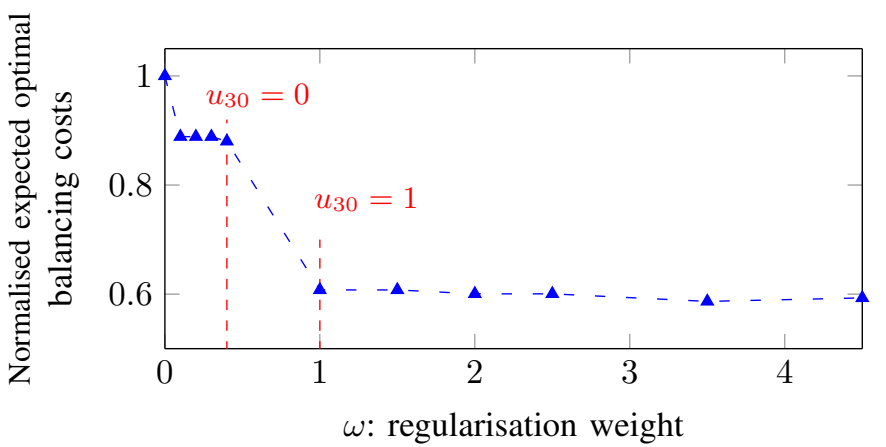

Fig. 3. Optimal exp. balancing costs for varying the regularisation weight $\omega$.

of the mean. This simplification allows us to evaluate the impact of mis-estimation on the overall day-ahead schedule and real-time dispatch, a critical point of assessment for any application of stochastic programming. In this context, in Fig. 2 we demonstrate the differences between the perfect and imperfect estimates by plotting the cumulative distribution function of the Beta distribution with the parameters used in the simulation, alongside with the distributions that correspond to over and under estimating of the mean of the actual Beta distribution. As expected, the whole shape of the distribution is affected by the mis-estimation of the mean. Equations 15 give the relationship between $\alpha, \beta$ and the mean.

Fig. 3 shows the normalised optimal balancing costs while varying the weighting $\omega$ on the CVaR risk measure. When the weighting is zero, the model does not take into account the costs introduced by the mis-estimation of wind. We observe that as the weighting increases, the balancing cost decreases. More conventional generation is scheduled in day ahead market to minimise the mis-estimation in real time. Note that for $0 \leq \omega \leq 0.4$ generator at bus 30 (most expensive generator) is not committed. But for values $\omega>0.4$ generator at bus 30 is committed. The absence of the risk measure defined by CVaR (i.e. $\omega=0$ ), models a risk neutral policy of committing the generators. However with a non-zero weight on the risk measure we model a more robust and risk-aware policy.

Following the preceding analysis, we study the computational tractability of the proposed model, by plotting the optimal cost with respect to the number of scenarios. In Fig. 4 the solid red line represents the generation cost of conventional generators with the dashed blue line showing the expected optimal balancing cost. We observe that the estimated balancing costs are zero in the deterministic case: when only one scenario is considered, noting that the deterministic case corresponds to the model with perfect information about the future realisations. We further observe that the expected balancing costs increase with the increase in number of scenarios. However the day-ahead generation cost has a very steady behaviour after 20 scenarios. This indicates that after 20 scenario adding more scenarios does not have a severe influence on the generation cost.

Finally, we investigate the impact of imperfect information by analysing the wind power producer at bus 34 . Fig. 5 shows 


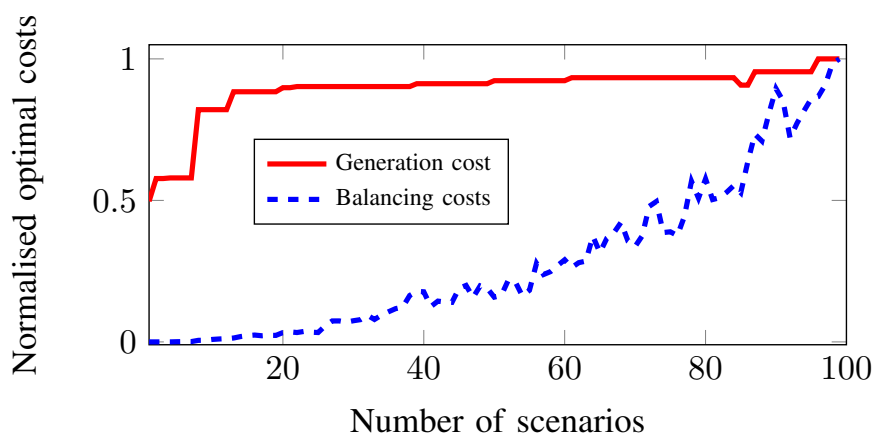

Fig. 4. Optimal cost corresponding to number of scenarios.

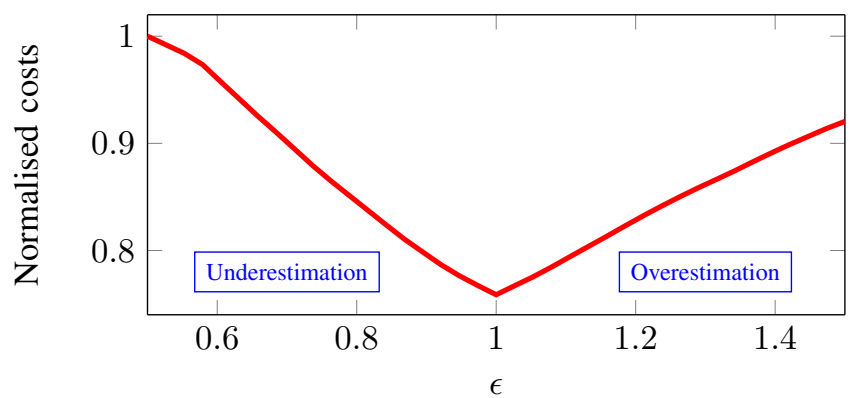

Fig. 5. Effect of mis-estimation of wind power generation on overall costs.

that both over and under estimation result in a significant increase of the overall cost (i.e. balancing costs plus generation costs). We observe that under-estimation costs are as high as $25 \%$ while over-estimation costs are as high as $15 \%$. In the first case, the commitment of expensive generators in maintaining the demand and generation balance leads to increase in generation costs, while for over-estimation generators face additional costs in order to buy surplus from real time market, hence higher balancing costs.

\section{CONCLUSION AND FUTURE WORK}

This paper solves a unit commitment problem for a dayahead market that can accept probabilistic offers based on a risk measure defined by the CVaR. We simulate this model on the a 39-bus problem and show that considerable benefits in terms of re-dispatch cost can be achieved by carefully managing the risk of mis-estimation. We have also illustrated that the mean of the distribution is an ideal candidate to plan the day-ahead operation of a system.

For future research, we intend to extend the proposed model so it captures more of the challenging aspects of a power system. As a starting point, the single-dimensional time framework will be extended so that the model can take into consideration inter-temporal constraints. In doing so this research can be extended to a multi-stage unit commitment model that considers the minimum up and down times of the generators. Furthermore, we intend to consider several misestimating wind power producers and take into account the correlation among different wind power producers. Finally, we intend to challenge the use of the mean as the optimal statistic for the clearing of the day-ahead market and examine the effect of different statics on the optimal solution.

\section{ACKNOWLEDGEMENT}

W. A. Bukhsh is supported by EU FP7 project 'GARPUR' under Grant Agreement No 608540. The other authors are partly supported by the Danish Council for Strategic Research (DSF) through the project '5s-Future Electricity Markets', No. 12-132636/DSF.

\section{REFERENCES}

[1] R. Billinton, Y. Gao, and R. Karki, "Application of a joint deterministicprobabilistic criterion to wind integrated bulk power system planning," Power Systems, IEEE Transactions on, vol. 25, no. 3, pp. 1384-1392, Aug 2010

[2] V. M. Zavala, K. Kim, M. Anitescu, and J. Birge, "A Stochastic Electricity Market Clearing Formulation with Consistent Pricing Properties," ArXiv e-prints, Oct. 2015.

[3] N. Zhang, C. Kang, Q. Xia, Y. Ding, Y. Huang, R. Sun, J. Huang, and J. Bai, "A convex model of risk-based unit commitment for day-ahead market clearing considering wind power uncertainty," Power Systems, IEEE Transactions on, vol. 30, no. 3, pp. 1582-1592, May 2015.

[4] A. Tuohy, P. Meibom, E. Denny, and M. O'Malley, "Unit commitment for systems with significant wind penetration," Power Systems, IEEE Transactions on, vol. 24, no. 2, pp. 592-601, May 2009.

[5] J. R. Birge and F. Louveaux, Introduction to Stochastic Programming. Springer, 1997.

[6] G. Pritchard, G. Zaker, and A. Philpott, "A single-settlement, energyonly electric power market for unpredictable and intermittent participants." Operations Research, vol. 58, pp. 1210-1219, 2010.

[7] J. M. Morales, A. J. Conejo, K. Liu, and J. Zhong, "Pricing electricity in pools with wind producers." IEEE Transactions On Power Systems, vol. 27, no. 3, pp. 1366-1376, 2012.

[8] T. Summers, J. Warrington, M. Morari, and J. Lygeros, "Stochastic optimal power flow based on conditional value at risk and distributional robustness," International Journal of Electrical Power \& Energy Systems, vol. 72, pp. 116 - 125, 2015, the Special Issue for 18th Power Systems Computation Conference.

[9] Y. Zhang and G. Giannakis, "Robust optimal power flow with wind integration using conditional value-at-risk," in Smart Grid Communications (SmartGridComm), 2013 IEEE International Conference on, Oct 2013, pp. 654-659.

[10] A. Papakonstantinou and P. Pinson, "Towards electricity markets accommodating uncertain offers." in Proceedings of 13th International Workshop on Large-Scale Integration of Wind Power and Transmission Networks, 2014

[11] P. Pinson, "Wind energy: Forecasting challenges for its operational management," Statistical Science, vol. 28, no. 4, pp. 564-585, 2013.

[12] C. Lowery and M. O'Malley, "Impact of wind forecast error statistics upon unit commitment," Sustainable Energy, IEEE Transactions on, vol. 3 , no. 4, pp. 760-768, Oct 2012.

[13] R. T. Rockafellar and S. Uryasev, "Conditional value-at-risk for general loss distributions," Journal of Banking and Finance, vol. 26, no. 7, pp. 1443-1471, 2002.

[14] J. Zhu, Optimization of Power System Operation. IEEE Press, 2009.

[15] "IBM ILOG CPLEX Optimizer," http://www01.ibm.com/software/integration/optimization/cplex-optimizer/, 2010.

[16] W. E. Hart, C. Laird, J.-P. Watson, and D. L. Woodruff, Pyomooptimization modeling in python. Springer Science \& Business Media, 2012, vol. 67.

[17] R. D. Zimmerman, C. E. Murillo-Sánchez, and R. J. Thomas, "Matpower: Steady-state operations, planning, and analysis tools for power systems research and education," IEEE Transactions on Power Systems, vol. 26, pp. 12-19, 2011.

[18] C. Ferreira, F. Barbosa, and C. Agreira, "Transient stability preventive control of an electric power system using a hybrid method," in Power System Conference, 2008. MEPCON 2008. 12th International MiddleEast, March 2008, pp. 141-145.

[19] J. M. Morales, M. Zugno, S. Pineda, and P. Pinson, "Electricity market clearing with improved scheduling of stochastic production," European Journal of Operational Research, vol. 235, no. 3, pp. 765-774, 2014.

[20] X. Liu, "Impact of beta-distributed wind power on economic load dispatch," Electric Power Components and Systems, vol. 39, pp. 768779, 2011. 